\title{
Quantum graphs with the Bethe-Sommerfeld property
}

\author{
P. Exner ${ }^{1,2}$, O. Turek ${ }^{2,3,4}$ \\ ${ }^{1}$ Doppler Institute for Mathematical Physics and Applied Mathematics, \\ Czech Technical University, Břehová 7, 11519 Prague, Czechia \\ ${ }^{2}$ Department of Theoretical Physics, Nuclear Physics Institute CAS, \\ 25068 Řež near Prague, Czech Republic \\ ${ }^{3}$ Bogoliubov Laboratory of Theoretical Physics, Joint Institute \\ for Nuclear Research, 141980 Dubna, Russia \\ ${ }^{4}$ Laboratory for Unified Quantum Devices, Kochi University \\ of Technology, Kochi 782-8502, Japan \\ exner@ujf.cas.cz,o.turek@ujf.cas.cz
}

\begin{abstract}
In contrast to the usual quantum systems which have at most a finite number of open spectral gaps if they are periodic in more than one direction, periodic quantum graphs may have gaps arbitrarily high in the spectrum. This property of graph Hamiltonians, being generic in a sense, inspires the question about the existence of graphs with a finite and nonzero number of spectral gaps. We show that the answer depends on the vertex couplings together with commensurability of the graph edges. A finite and nonzero number of gaps is excluded for graphs with scale invariant couplings; on the other hand, we demonstrate that graphs featuring a finite nonzero number of gaps do exist, illustrating the claim on the example of a rectangular lattice with a suitably tuned $\delta$-coupling at the vertices.
\end{abstract}

Keywords: periodic quantum graphs, gap number, $\delta$-coupling, rectangular lattice graph, scale-invariant coupling, Bethe-Sommerfeld conjecture, golden mean.

Quantum graphs [1] have attracted much attention both from the practical point of view as models of nanostructures as well as a tool to study the properties of quantum systems with a nontrivial topology of the configuration space. The topological richness of quantum graphs allows them to exhibit properties different from those of the 'usual' quantum Hamiltonians; examples are well known, for instance, the existence of compactly supported eigenfunctions on infinite graphs [1, Sec. 3.4] or the possibility of having flat bands only as is the case for magnetic chain graphs with a half-of-the-quantum flux through each chain element [2].

In this letter, we are going to consider another situation where quantum graphs are known to behave unusually. Our problem concerns the gap structure of the spectrum of periodic quantum graphs. Recall that the finiteness of the open gap number for periodic quantum systems in dimension two or more was conjectured in the early days of quantum theory by Bethe and Sommerfeld [3]. The validity of the conjecture was taken for granted even if its proof turned out to pose a mathematically difficult problem. It took a long time before it was rigorously established for the 'usual' periodic Schrödinger operators [4-8]. Nevertheless, the situation appears to be different for quantum graphs, as we will see below.

The traditional reasoning behind the Bethe-Sommerfeld conjecture relies on the behavior of the spectral bands identified with the ranges of the dispersion curves or surfaces which, in contrast to the one-dimensional situation, typically overlap, making opening of gaps more and more difficult as we proceed to higher energies. The situation with graphs might be similar [1, Sec. 4.7] but the spectral behavior need not be the same, one reason being the possibility of resonant gaps. The existence of gaps coming from a graph decoration was first observed in the discrete graph context [9] and the effect is present for metric graphs as well [1, Sec. 5.1]. In addition, the recently discovered universality property of periodic graphs [10], valid in the generic situation when the graph edges are incommensurate and the vertex coupling is the simplest possible, usually called Kirchhoff, shows that the occurrence of infinitely many open gaps is quite typical.

This prompts one to ask whether there are quantum graphs with the band spectrum similar to that of the 'usual' multidimensional periodic systems, i.e. a nonzero and finite number of open gaps; for the sake of brevity, we shall speak of the Bethe-Sommerfeld property. With this question in mind, our aim in this letter is twofold. On the one hand we will show that the answer depends on the vertex coupling and there are classes of couplings for which such a behavior is excluded. On the other hand, using a simple example we shall demonstrate that periodic graphs with Bethe-Sommerfeld property do exist. 
Consider an infinite graph $\Gamma$ periodic in $\nu$ directions, with a slight abuse of notation we will speak of a $\mathbb{Z}^{\nu}$-periodicity, $\nu \geq 2$. The Hamiltonian is supposed to act as $-\mathrm{d}^{2} / \mathrm{d} x^{2}$ on each edge; to make it a self-adjoint operator, one has to impose appropriate coupling conditions at each vertex. The most general form of them [11,12] is $(U-I) \Psi+\mathrm{i}(U+I) \Psi^{\prime}=0$, where $\Psi, \Psi^{\prime}$ are vectors of the function and derivative values at the vertex, respectively, and $U$ is a unitary $n \times n$ matrix for $n$ denoting the degree of the vertex. According to the eigenvalues of $U$ one can split the coupling into the Dirichlet, Neumann, and Robin parts [1, Sec. 1.4]. Equivalently, the coupling can be written in so-called ST-form [13],

$$
\left(\begin{array}{cc}
I^{(r)} & T \\
0 & 0
\end{array}\right) \Psi^{\prime}=\left(\begin{array}{cc}
S & 0 \\
-T^{*} & I^{(n-r)}
\end{array}\right) \Psi
$$

for certain $r, S$, and $T$, where $I^{(r)}$ is the identity matrix of order $r$ and $S$ is a Hermitian matrix that refers to the Robin part. A coupling is called scale-invariant if the matrix $U$ has no eigenvalues other than \pm 1 ; it is easy to see that this happens if and only if $S=0$ [14]. Recall that the on-shell scattering matrix $\mathcal{S}(k)$ for the vertex in question is in the $S T$-formalism given by

$$
\mathcal{S}(k)=-I^{(n)}+2\left(\begin{array}{c}
I^{(r)} \\
T^{*}
\end{array}\right)\left(I^{(r)}+T T^{*}-\frac{1}{\mathrm{i} k} S\right)^{-1}\left(\begin{array}{ll}
I^{(r)} & T
\end{array}\right)
$$

and it is obvious that $\mathcal{S}(k)$ is independent of $k$ iff $S=0$.

The spectrum is obtained using the Bloch-Floquet theory [1, Sec. 4.2]. We cut from $\Gamma$ its elementary cell $\Gamma_{\text {per }}$ which is assumed to be a finite graph with a family of pairs of 'antipodal' vertices related mutually by the conditions $\psi\left(v_{+}\right)=\mathrm{e}^{\mathrm{i} \vartheta_{l}} \psi\left(v_{-}\right)$and $\psi^{\prime}\left(v_{+}\right)=\mathrm{e}^{\mathrm{i} \vartheta_{l}} \psi^{\prime}\left(v_{-}\right)$with some $\vartheta_{l} \in(-\pi, \pi]$, where $l=1, \ldots, \nu$ with $\nu$ being the dimension of translation group associated with graph periodicity; the pair of edges with the endpoints $v_{ \pm}$can be turned into a single edge by identifying these endpoints, and the acquired phase $\vartheta_{l}$ coming from the Bloch conditions can be also regarded as being induced by a suitable magnetic potential.

The spectral problem can be solved in the usual way, cf. [1, Sec. 2.1] or [10]. Assuming that $\Gamma_{\text {per }}$ has $E$ edges, we consider three $2 E \times 2 E$ matrices. The diagonal matrix $\mathbf{L}$ is determined by the lengths of the directed edges (bonds) of $\Gamma_{\text {per }}$, the diagonal matrix $\mathbf{A}$ has the entries $\mathrm{e}^{\mathrm{i} \vartheta_{l}}$ or $\mathrm{e}^{-\mathrm{i} \vartheta_{l}}$ at the positions corresponding to the edges created by the mentioned vertex identification, and all its other entries are zero, and finally, the matrix $\mathbf{S}$ is the bond scattering matrix, which contains directed edge-to-edge scattering coefficients. Using them, we define

$$
F(k ; \vec{\vartheta}):=\operatorname{det}\left(\mathbf{I}-\mathrm{e}^{\mathrm{i}(\mathbf{A}+k \mathbf{L})} \mathbf{S}(k)\right) ;
$$

then $k^{2} \in \sigma(H)$ holds iff there is a $\vartheta \in(-\pi, \pi]^{\nu}$ such that the secular equation $F(k ; \vec{\vartheta})=0$ is satisfied.

Suppose now that the couplings at all the vertices of $\Gamma$ are scale-invariant. This, in particular, means the matrix S entering formula (2) is independent of $k$, hence the value $F(k ; \vec{\vartheta})$ depends on the vectors $\vec{\vartheta}$ and $k \ell_{0}, k \ell_{1}, \ldots, k \ell_{d}$, where $\left\{\ell_{0}, \ell_{1}, \ldots, \ell_{d}\right\}, d \leq E-1$, is the set of mutually different edge lengths of $\Gamma$, and $F(k ; \vec{\vartheta})$ is obviously $2 \pi$-periodic in the terms $k \ell_{0}, k \ell_{1}, \ldots, k \ell_{d}$. The secular equation can be then written as

$$
F\left(\left\{k \ell_{0}\right\}_{(2 \pi)},\left\{k \ell_{1}\right\}_{(2 \pi)}, \ldots,\left\{k \ell_{d}\right\}_{(2 \pi)} ; \vec{\vartheta}\right)=0,
$$

where $\{x\}_{(2 \pi)}:=2 \pi\{x /(2 \pi)\}$, for $\{\cdot\}$ denoting the difference between the number and its nearest integer. This allows us to prove that

(i) if $\sigma(H)$ has a gap, then it has infinitely many gaps,

(ii) the gaps can be divided into series with asymptotically constant lengths with respect to $k$, and

(iii) in particular, if all the edge lengths are commensurate, the momentum spectrum is periodic.

The easiest part to verify is (iii). In that case there is an elementary length $L>0$ and integers $m_{j} \in \mathbb{N}$ such that $\ell_{j}=m_{j} L$ holds for $j=0,1, \ldots, d$, and consequently, the left-hand side of (3) is $2 \pi / L$-periodic with respect to $k$. Parts (i) and (ii) are more involved and we just sketch the argument referring to [15] for the full proof.

To prove (i) we consider a $k>0$ satisfying $k^{2} \notin \sigma(H)$ and use it to prove the existence, for any given $C>0$, a $k^{\prime}>C$ such that $\left(k^{\prime}\right)^{2} \notin \sigma(H)$. Due to the continuity of $F$, it is sufficient to find a $k^{\prime}$ so that the values $k^{\prime} \ell_{j}$ are arbitrarily close to $k \ell_{j}$ up to an integer multiple of $2 \pi$. To this aim, we denote $\alpha_{j}=\ell_{j} / \ell_{0}$ and employ the simultaneous version of the Dirichlet's approximation theorem by which for any $N \in \mathbb{N}$ there are integers $p_{1}, \ldots, p_{d}, q \in \mathbb{Z}, 1 \leq q \leq N$, such that

$$
\left|\alpha_{j}-\frac{p_{j}}{q}\right| \leq \frac{1}{q N^{1 / d}}
$$


Choosing integers $m>\ell_{0} C / 2 \pi$ and $N>(2 \pi m / \delta)^{d}$, and putting $k_{\delta}^{\prime}:=k+2 \pi m q / \ell_{0}$, it is straightforward to see that $k_{\delta}^{\prime}>C$ and, using (4), to check that $\left|\left\{k_{\delta}^{\prime} \ell_{j}-k \ell_{j}\right\}_{(2 \pi)}\right|<\delta$ for all $j$. Moreover, the latter inequality in combination with the argument used to prove (i) yields the claim (ii).

In fact, one can exclude the Bethe-Sommerfeld property for a wider class of graphs. Given a vertex coupling described by condition (1), we consider the associated scale-invariant one obtained by replacing the Robin part $S$ by zero. The vertex scattering matrix can be then written as $\mathcal{S}(k)=\mathcal{S}_{0}+\mathcal{S}_{1}(k) / k$, where $\mathcal{S}_{0}$ is the scattering matrix of the associated scale-invariant vertex coupling. Then the function $F(k ; \vec{\vartheta})$ in the secular equation is

$$
F_{0}\left(\left\{k \ell_{0}\right\}_{(2 \pi)},\left\{k \ell_{1}\right\}_{(2 \pi)}, \ldots,\left\{k \ell_{d}\right\}_{(2 \pi)} ; \vec{\vartheta}\right)+\frac{1}{k} F_{1}(k ; \vec{\vartheta}),
$$

where the subscript zero at $F_{0}$ refers to the Hamiltonian $H_{0}$ of the graph in which all the couplings have been replaced by the associated scale-invariant ones. Using the fact that the leading behavior of $F(\cdot ; \vec{\vartheta})$ at high energies comes from the scale-invariant term, it is not difficult to see that [15]

(i) if $\sigma\left(H_{0}\right)$ has an open gap, then $\sigma(H)$ has infinitely many gaps,

(ii) if all the edge lengths of $\Gamma$ are commensurate, then the gaps of $\sigma(H)$ asymptotically coincide with those of $\sigma\left(H_{0}\right)$.

Let us turn to our second topic, the existence of graphs with the Bethe-Sommerfeld property. To this goal, we revisit the lattice Kronig-Penney model introduced in [16] and further discussed in [17,18]. In this case, $\Gamma$ is a rectangular lattice graph in the plane with edges of lengths $a$ and $b$, cf. Fig. 1.

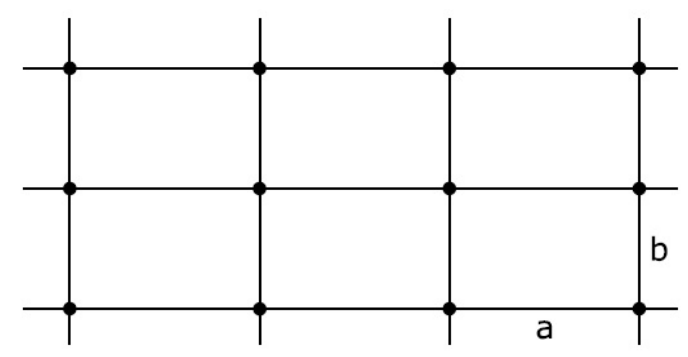

FIG. 1. The rectangular-lattice graph

The Hamiltonian $H=H_{\alpha, a / b}$ is the negative Laplacian with the $\delta$ coupling condition in each vertex, i.e. the functions are continuous there and satisfy $\sum_{j=1}^{4} \psi^{\prime}(v)=\alpha \psi(v)$ with a fixed parameter $\alpha \in \mathbb{R}$.

According to [17], a number $k^{2}>0$ belongs to a gap if and only if $k>0$ satisfies the gap condition, which reads

$$
\tan \left(\frac{k a}{2}-\frac{\pi}{2}\left\lfloor\frac{k a}{\pi}\right\rfloor\right)+\tan \left(\frac{k b}{2}-\frac{\pi}{2}\left\lfloor\frac{k b}{\pi}\right\rfloor\right)<\frac{|\alpha|}{2 k}
$$

for $\alpha>0$ and the analogous one with tan replaced by cot if $\alpha<0$; we neglect the case $\alpha=0$ where the spectrum is trivial, $\sigma(H)=[0, \infty)$. For $\alpha<0$ the spectrum extends to the negative part of the real axis and may have a gap there, but since such a gap always has a positive part [18], we may restrict our attention to examining gaps in the positive spectrum.

The crucial quantity is the ratio $\theta=a / b$. It is obvious that $\sigma(H)$ has infinitely many gaps once $\alpha \neq 0$ and $\theta$ is rational, and the same is true for the 'well approximable' irrationals [17]. We thus focus on the other irrationals, called badly approximable, i.e those to which there is a $c>0$ such that

$$
\left|\theta-\frac{p}{q}\right|>\frac{c}{q^{2}}
$$

for all $p, q \in \mathbb{Z}$ with $q \neq 0$. These numbers form a set of zero Lebesgue measure. Alternatively they can be characterized as irrationals for which the sequence in the continued-fraction representation, $\theta=\left[c_{0} ; c_{1}, c_{2}, \ldots\right]$, is bounded [19], or as numbers whose Markov constant $\mu(\theta)$, defined [20] for $\theta \in \mathbb{R}$ as

$$
\mu(\theta)=\inf \left\{c>0 \mid\left(\exists_{\infty}(p, q) \in \mathbb{N}^{2}\right)\left(\left|\theta-\frac{p}{q}\right|<\frac{c}{q^{2}}\right)\right\},
$$

is strictly positive. It is convenient to introduce a one-sided analog $v(\theta)$ of the Markov constant, with the last inequality in (6) replaced by $0<\theta-p / q<c / q^{2}$. We have $\mu(\theta)=\min \left\{v(\theta), v\left(\theta^{-1}\right)\right\}$; the number $v(\theta)$ may or may not coincide with the Markov constant [21]. 
To obtain the existence claim, we focus on the situation where $\theta$ is the 'worst approximable' irrational, the golden mean, $\phi=(\sqrt{5}+1) / 2$. Our result about the golden-mean lattice is the following:

(i) If $\alpha>\pi^{2} /(\sqrt{5} a)$ or $\alpha \leq-\pi^{2} /(\sqrt{5} a)$, the spectrum has infinitely many gaps.

(ii) If $-2 \pi / a \tan ((3-\sqrt{5}) \pi / 4) \leq \alpha \leq \pi^{2} /(\sqrt{5} a)$ there are no gaps in the spectrum.

(iii) If $-\pi^{2} /(\sqrt{5} a)<\alpha<-2 \pi / a \tan ((3-\sqrt{5}) \pi / 4)$, there is a nonzero and finite number of gaps in the spectrum.

(iv) Moreover, put $A_{j}:=2 \pi\left(\phi^{2 j}-\phi^{-2 j}\right) / \sqrt{5} \tan \left(\pi \phi^{-2 j} / 2\right)$, then there are exactly $N$ gaps in the spectrum if $-A_{N+1} \leq \alpha<-A_{N}$.

Note that the window in which Bethe-Sommerfeld property occurs in this example (statement (iii)), is rather narrow, roughly $4.298 \lesssim-\alpha a \lesssim 4.414$.

The proof of these claims is rather involved and we limit ourselves with mentioning its key elements referring to [15] for the full exposition. The central notion is that of the Diophantine approximation of third type from below (from above, respectively). The former is a number $p / q$ with $p, q \in \mathbb{Z}$ such that

$$
0<q(q \theta-p)<q^{\prime}\left(q^{\prime} \theta-p^{\prime}\right)
$$

holds for all $p^{\prime} / q^{\prime} \geq \theta$ with $p^{\prime} / q^{\prime} \neq p / q, p^{\prime}, q^{\prime} \in \mathbb{Z}$ and $0<q^{\prime} \leq q$; the approximation from above has (7) replaced by $0<q(p-q \theta)<q^{\prime}\left(p^{\prime}-q^{\prime} \theta\right)$. We note that $v(\theta)$ is the infimum of those $q(q \theta-p)$ for which $p / q$ is a best approximation from below to $\theta$. These approximations are also closely related to convergents obtained from truncated continued-fraction representation of $\theta$. Specifically, every best approximation of the third kind from below to a $\theta \in \mathbb{R}$ is a convergent of $\theta$, and on the other hand, every best approximation from above is either $\lceil\theta\rceil$ or a convergent of $\theta$, where $\lceil\cdot\rceil$ is the ceiling function.

The described Diophantine approximation in combination with the gap condition allows us to estimate the number of gaps for a given ratio $\theta=a / b$ and coupling parameter $\alpha$. This has to be done for each sign of $\alpha$ separately. If $\alpha>0$, condition (5) yields that

- if $\alpha<\pi^{2} \cdot \min \left\{v(\theta) / b, v\left(\theta^{-1}\right) / a\right\}$, the spectrum has at most finitely many gaps. If the opposite (sharp) inequality holds true, the number of gaps is infinite;

- if $\alpha \leq \gamma_{+}$for $\gamma_{+}$given by

$$
\gamma_{+}:=\min _{\eta=\theta, \theta^{-1}} \inf _{m \in \mathbb{N}}\left\{2 \pi m \sqrt{\frac{\eta}{a b}} \tan \left(\frac{\pi}{2}(m \eta-\lfloor m \eta\rfloor)\right)\right\},
$$

the spectrum has no gaps. If $\alpha>\gamma_{+}$, there are gaps in the spectrum;

- in particular, if $\gamma_{+}<\alpha<\pi^{2} \cdot \min \left\{v(\theta) / b, v\left(\theta^{-1}\right) / a\right\}$, there is a nonzero and finite number of gaps in the spectrum.

The golden mean $\phi$ in our example has continued-fraction representation $\phi=[1 ; 1,1, \ldots]$, therefore, its convergents are ratios $F_{n+1} / F_{n}$ of the Fibonnaci numbers $F_{n}=\left(\phi^{n}-(-\phi)^{-n}\right) / \sqrt{5}$, and we have $\mu(\phi)=v(\phi)=1 / \sqrt{5}$ in view of the Hurwitz theorem [22]. Hence, we get

$$
\gamma_{+}=\frac{\pi^{2}}{\sqrt{5} a} \quad \text { and } \quad \pi^{2} \cdot \min \left\{\frac{v(\phi)}{b}, \frac{v\left(\phi^{-1}\right)}{a}\right\}=\frac{\pi^{2}}{\sqrt{5} a},
$$

which implies for all positive $\alpha$ either infinite number of spectral gaps or none at all. Alternatively, for $\alpha<0$ the gap condition implies that

- if $|\alpha|<\pi^{2} \cdot \min \left\{v(\theta) / a, v\left(\theta^{-1}\right) / b\right\}$, the number of gaps in the positive spectrum is at most finite. By contrast, for $|\alpha|$ greater than the right-hand side of the above inequality, there are infinitely many spectral gaps, while

- if $|\alpha| \leq \gamma_{-}$for $\gamma_{-}$given by the relation analogous to (8) in which $m \eta-\lfloor m \eta\rfloor$ is replaced by $\lceil m \eta\rceil-m \eta$, the spectrum has no gaps. If $|\alpha|>\gamma_{-}$, there are gaps in the spectrum;

- in particular, if $\gamma_{-}<|\alpha|<\pi^{2} \cdot \min \left\{v(\theta) / a, v\left(\theta^{-1}\right) / b\right\}$, there is a nonzero and finite number of gaps in the spectrum.

The last of these results, together with the easily verifiable formula

$$
\gamma_{-}=\frac{2 \pi}{a} \tan \frac{(3-\sqrt{5}) \pi}{4}
$$


proves the claim (iii) above, demonstrating thus the existence of graphs with the Bethe-Sommerfeld property. To prove (iv), the first step consists in showing that the number of gaps in the golden-mean lattice graph is equal to the number of solutions $m \in \mathbb{N}$ of

$$
\frac{2 \pi m}{a} \tan \left(\frac{\pi}{2}(\lceil m \phi\rceil-m \phi)\right)<|\alpha|
$$

If $-A_{N+1} \leq \alpha<-A_{N}$, one can verify that the inequality is satisfied only for $m=F_{n}$ with $n=2,4,6, \ldots, 2 N$; this implies the existence of exactly $N$ gaps.

Since a finite nonzero number of gaps occurred in the above example only for attractive vertex couplings, it is natural to ask whether the attractivity of the coupling is always a necessary condition for the Bethe-Sommerfeld property. It appears that it is not the case, a more thorough analysis of the gap condition [15] shows that, for instance, the edge ratio

$$
\theta=\frac{2 t^{3}-2 t^{2}-1+\sqrt{5}}{2\left(t^{4}-t^{3}+t^{2}-t+1\right)} \quad \text { with } t \in \mathbb{N}, t \geq 3
$$

which has the continued-fraction representation $[0 ; t, t, 1,1,1,1, \ldots]$, yields the lattice graph spectrum with the said property for a certain $\alpha>0$ and for a certain $\alpha<0$ as well. This observation can be stated more generally [15] and allows one to explicitly construct ratios to achieve the Bethe-Sommerfeld property.

In conclusion, we have proved and demonstrated on concrete examples that there are periodic quantum graphs, the spectrum of which contains a nonzero and finite number of open gaps. We have also described how this property depends on the type of the vertex coupling; in particular, we showed that a quantum graph cannot be of the Bethe-Sommerfeld type if its couplings are scale invariant or associated with scale-invariant ones.

\section{Acknowledgements}

The research was supported by the Czech Science Foundation (GAČR) within the project 17-01706S.

\section{References}

[1] Berkolaiko G., Kuchment P. Introduction to Quantum Graphs. AMS, Providence, R.I., 2013.

[2] Exner P., Manko S. S. Spectra of magnetic chain graphs: coupling constant perturbations. J. Phys. A: Math. Theor., 2015, 48, P. 125302.

[3] Sommerfeld A., Bethe H. Electronentheorie der Metalle. 2nd ed., Handbuch der Physik, Springer Verlag, Berlin, 1933.

[4] Skriganov M. M. Proof of the Bethe-Sommerfeld conjecture in dimension two. Soviet Math. Dokl., 1979, 20, P. 956-959.

[5] Dahlberg J., Trubowitz E. A remark on two dimensional periodic potentials. Comment. Math. Helvetici, 1982, 57, P. 130-134.

[6] Skriganov M. M. The spectrum band structure of the threedimensional Schrödinger operator with periodic potential. Invent. Math., 1985, 80, P. 107-121.

[7] Helffer B., Mohamed A. Asymptotic of the density of states for the Schrödinger operator with periodic electric potential. Duke Math. J., 1998, 92, P. 1-60

[8] Parnovski L. Bethe-Sommerfeld conjecture. Ann. Henri Poincaré, 2008, 9, P. 457-508.

[9] Schenker J. H., Aizenman M. The creation of spectral gaps by graph decoration. Lett. Math. Phys., 2000, 53, P. $253-262$.

[10] Band R., Berkolaiko G. Universality of the momentum band density of periodic networks. Phys. Rev. Lett., 2013,111, P. 130404.

[11] Kostrykin V., Schrader R. Kirchhoff's rule for quantum wires. J. Phys. A: Math. Gen., 1999, 32, P. 595-620.

[12] Harmer M. Hermitian symplectic geometry and extension theory. J. Phys. A: Math. Gen., 2000, 33, P. $9193-9203$.

[13] Cheon T., Exner P., Turek O. Approximation of a general singular vertex coupling in quantum graphs. Ann. Phys. (NY), 2010, 325, P. 548-578.

[14] Cheon T., Exner P., Turek O. Tripartite connection condition for a quantum graph vertex. Phys. Lett. A, 2010, 375, P. 113-118.

[15] Exner P., Turek O. Periodic quantum graphs from the Bethe-Sommerfeld perspective, arXiv: 1705.07306 [math-ph ]

[16] Exner P. Lattice Kronig-Penney models. Phys. Rev. Lett. 1995, 74, P. 3503-3506.

[17] Exner P. Contact interactions on graph superlattices. J. Phys. A: Math. Gen., 1996, 29, P. 87-102.

[18] Exner P., Gawlista R. Band spectra of rectangular graph superlattices. Phys. Rev. B, 1996, 53, P. $7275-7286$.

[19] Khinchin Y. Ya. Continued Fractions. University of Chicago Press, 1964.

[20] Cassels J. W. An introduction to Diophantine Approximation. Cambridge University Press, 1957.

[21] Pelantová E., Starosta Š., Znojil M. Markov constant and quantum instabilities. J. Phys. A: Math. Theor., 2016,49 , P. 155201.

[22] Hurwitz A. Über die angenäherte Darstellung der Irrationalzahlen durch rationale Brüche. Math. Ann., 1891, 39, P. 279-284. 